\title{
The period lengths of inversive congruential recursions
}

\author{
by \\ Wun-Seng Chou (Taipei)
}

1. Introduction. Let $p$ be a prime. For fixed elements $a$ and $b$ of the finite field $\mathrm{GF}(p)=\mathbb{Z} / p \mathbb{Z}$ (it can be identified with the set $Z_{p}=$ $\{0,1, \ldots, p-1\}$ together with the operations of addition and multiplication modulo $p$ ), Eichenauer and Lehn [3] defined sequences $X\left(x_{0} ; a, b\right): x_{0}, x_{1}, \ldots$ by choosing initial elements $x_{0} \in \mathrm{GF}(p)$ and using the recursion

$$
\begin{aligned}
& x_{n+1}=a x_{n}^{-1}+b \quad \text { if } x_{n} \neq 0, \quad \text { for all } n \geq 0 . \\
& x_{n+1}=b \quad \text { if } x_{n}=0
\end{aligned}
$$

They used this method as a nonlinear method to generate pseudorandom numbers. Niederreiter [10] generalized it over arbitrary finite field $\operatorname{GF}(q)$ when he studied pseudorandom vectors. See also Eichenauer-Herrmann [5] and Niederreiter [11, Chapters 8 and 10] and [12] for more details on these methods. Because the recursion (1) is used to construct pseudorandom numbers and pseudorandom vectors, the problem of when the sequence $X\left(x_{0} ; a, b\right)$ has the maximal period length has been studied intensively: see, for instance, Chou [1], Eichenauer and Lehn [3], Flahive and Niederreiter [8], and Niederreiter [12].

For studying pseudorandom numbers with modulus a composite positive integer $m$, the recursion (1) must be changed into the following: For all $n \geq 0$,

$$
x_{n+1} \equiv a x_{n}^{-1}+b \bmod m \quad \text { provided } \operatorname{gcd}\left(x_{n}, m\right)=1 .
$$

So, every term of $X\left(x_{0} ; a, b\right)$ must be relatively prime to $m$. If $m=p_{1}^{r_{1}} \ldots p_{t}^{r_{t}}$, where $t \geq 2$ and $p_{1}, \ldots, p_{t}$ are distinct primes, is the prime factorization of $m$, then the period length of $X\left(x_{0} ; a, b\right)$ with modulus $m$ equals the least common multiple of period lengths of $X\left(x_{0} ; a, b\right)$ with modulus $p_{i}^{r_{i}}, 1 \leq i$ $\leq t$. So, for studying the period length of $X\left(x_{0} ; a, b\right)$ with modulus $m$, it suffices to consider $X\left(x_{0} ; a, b\right)$ with modulus a prime power $p^{k}$. Eichenauer, Lehn, and Topuzoğlu [4] studied the maximal period length with modulus $2^{k}$. Since the sequence $X\left(x_{0} ; a, b\right)$ with modulus a prime divisor $p$ of $m$ instead of $m$ itself does not contain 0 modulo $p, X\left(x_{0} ; a, b\right)$ with modulus $p$ does not 
have the maximal period length. It is necessary to study all possible period lengths of the recursion (1) over $\operatorname{GF}(p)$. Eichenauer and Lehn [3] obtained some results on the period length of $X\left(x_{0} ; a, b\right)$ with prime modulus. Chou [2] generalized it over finite fields and got all possible period lengths of the sequence $X\left(x_{0} ; a, b\right)$. Also, Eichenauer-Herrmann [6], Eichenauer-Herrmann and Topuzoğlu [7] and Huber [9] studied the period length of $X\left(x_{0} ; a, b\right)$ with modulus any prime power.

As we have mentioned above, if the sequence $X\left(x_{0} ; a, b\right)$ is generated by the recursion $(2)$, the sequence $X\left(x_{0} ; a, b\right)$ with modulus $p$ does not have the maximal period length. To make up for this deficiency, Huber [9] suggested to consider the recursion

$$
x_{n+1} \equiv a x_{n}^{\phi(m)-1}+b \bmod m \quad \text { for all } n \geq 0,
$$

where $\phi(m)$ is Euler's totient function. This recursion is equivalent to the recursion (1) when $m$ is a prime number and equivalent to the recursion (2) whenever each term of the sequence $X\left(x_{0} ; a, b\right)$ with modulus $m$ is relatively prime to $m$. But the recursion (3) allows any term $x_{n}$ of $X\left(x_{0} ; a, b\right)$ and $m$ to have a common divisor greater than 1 . Huber [9] showed that if $m$ is square free, then $X\left(x_{0} ; a, b\right)$ has the maximal period length with modulus $m$ if and only if $X\left(x_{0} ; a, b\right)$ with modulus each prime divisor $p$ of $m$ has the period length $p$.

In this paper, we are going to describe all possible period lengths of sequences $X\left(x_{0} ; a, b\right)$ generated by each of recursions (2) and (3). For this purpose, we need the following results from Chou [2].

LEMMA 1. Let $p$ be a prime and let $x_{0}$, $a$ and $b$ be elements of the finite field $\operatorname{GF}(p)$. Let $X\left(x_{0} ; a, b\right)$ be the sequence obtained by taking the initial element $x_{0} \in \mathrm{GF}(p)$ and using the recursion (1). Let $f(x)=x^{2}-b x-a$ and let $\mathfrak{o}\left(m_{f}\right)$ be the order of the polynomial $m_{f}(x)=x^{2}+\left(b^{2} / a+2\right) x+1$ provided $a \neq 0$. Moreover, let $L\left(x_{0} ; a, b ; p\right)$ be the period length of $X\left(x_{0} ; a, b\right)$.

(A) If $a=0$, then $x_{n}=b$ for all $n \geq 1$ and so $L\left(x_{0} ; 0, b ; p\right)=1$.

(B) If $a \neq 0$ and $b=0=x_{0}$, then $x_{n}=0$ for all $n \geq 0$, and so $L(0 ; a, 0 ; p)=1$.

(C) If $a x_{0} \neq 0, a=x_{0}^{2}$ and $b=0$, then $x_{n}=x_{0}$ for all $n \geq 0$, and so $L\left(x_{0} ; a, 0 ; p\right)=1$.

(D) If $a x_{0} \neq 0, a \neq x_{0}^{2}$ and $b=0$, then $x_{n+2}=x_{n}$ and $x_{n+1} \neq x_{n}$ for all $n \geq 0$, and so $L\left(x_{0} ; a, 0 ; p\right)=2$. Then

(E) Let $f(x)=(x-\alpha)^{2}$ for some $\alpha \in \mathrm{GF}(p)$ (or equivalently, $b^{2}+4 a=0$ ).

(1) $L(\alpha ; a, b ; \operatorname{GF}(p))=1$,

(2) if $x_{0} \neq \alpha$, then $X\left(x_{0} ; a, b\right)$ contains 0 and $L\left(x_{0} ; a, b ; p\right)=p-1$.

(F) Let $f(x)=(x-\alpha)(x-\beta)$ for some $\alpha \neq \beta \in \mathrm{GF}\left(p^{2}\right)$. 
(1) If $f\left(x_{0}\right)=0$, then $L\left(x_{0} ; a, b ; p\right)=1$.

(2) If $p \neq 2$ and $\mathfrak{o}\left(m_{f}\right)$ is even, then $X(b / 2 ; a, b)$ contains 0 and $L(b / 2 ; a, b ; p)=\mathfrak{o}\left(m_{f}\right)-1$.

(3) If $p \neq 2$ and $\mathfrak{o}\left(m_{f}\right)$ is odd, then $X(b / 2 ; a, b)$ does not contain 0 and $L(b / 2 ; a, b ; p)=\mathfrak{o}\left(m_{f}\right)$.

(4) If $f\left(x_{0}\right) \neq 0, x_{0} \neq b / 2$ whenever $p \neq 2$, and the order $\mathfrak{o}\left(M_{f}\right)$ of the polynomial $M_{f}(x)=x^{2}-\left(2+\left(b^{2}+4 a\right) / f\left(x_{0}\right)\right) x+1$ over $\mathrm{GF}(p)$ divides $\mathfrak{o}\left(m_{f}\right)$ (or equivalently, $M_{f}(x)$ divides $x^{\mathfrak{o}\left(m_{f}\right)}-1$ ), then $X\left(x_{0} ; a, b\right)$ contains 0 and $L\left(x_{0} ; a, b ; p\right)=\mathfrak{o}\left(m_{f}\right)-1$.

(5) If $f\left(x_{0}\right) \neq 0, x_{0} \neq b / 2$ for $p \neq 2$, and $\mathfrak{o}\left(M_{f}\right)$ does not divide $\mathfrak{o}\left(m_{f}\right)$, then $X\left(x_{0} ; a, b\right)$ does not contain 0 and $L\left(x_{0} ; a, b ; p\right)=$ $\mathfrak{o}\left(m_{f}\right)$.

Using this lemma, we are going to study all possible period lengths of sequences $X\left(x_{0} ; a, b\right)$ with modulus $m$ generated by the recursion (2) in Section 2 and all possible period lengths of sequences $X\left(x_{0} ; a, b\right)$ with modulus $m$ generated by the recursion (3) in Section 3.

2. Inversive congruential recursion. Let $m \geq 4$ be a fixed composite integer and let $m=p_{1}^{r_{1}} \ldots p_{t}^{r_{t}}$ be the prime factorization of $m$, where $t \geq 2$, $p_{1}, \ldots, p_{t}$ are distinct primes, and $r_{1}, \ldots, r_{t}$ are positive integers. For integers $a, b$, and $x_{0}$, let $X\left(x_{0} ; a, b\right)$ be the sequence defined by the recursion (2) if it can be defined. As we have mentioned, every term of $X\left(x_{0} ; a, b\right)$ must be relatively prime to $m$ and the period length $L\left(x_{0} ; a, b ; m\right)$ of $X\left(x_{0} ; a, b\right)$ with modulus $m$ equals the least common multiple of the period lengths $L\left(x_{0} ; a, b ; p_{i}^{r_{i}}\right)$ of $X\left(x_{0} ; a, b\right)$ with moduli $p_{i}^{r_{i}}, 1 \leq i \leq t$. We are going to consider first the sequence $X\left(x_{0} ; a, b\right)$ with modulus a prime power $p^{k}$ with $k \geq 2$. First, we have the following "well-defined" property.

LEMma 2. Let $p$ be a prime, and let $k, a, b$ and $x_{0}$ be integers with $k \geq 2$. Moreover, let $f(x)=x^{2}-b x-a$. Then $a, b$ and $x_{0}$ can be used to define an infinite sequence $X\left(x_{0} ; a, b\right)$ with modulus $p^{k}$ by the recursion (2) if and only if one of the following conditions holds:

(A) $a \equiv 0 \bmod p$ and $\operatorname{gcd}\left(b x_{0}, p\right)=1$.

(B) $\operatorname{gcd}\left(a x_{0}, p\right)=1$ and $b \equiv 0 \bmod p$.

(C) $\operatorname{gcd}\left(a b x_{0}, p\right)=1, b \equiv 2 x_{0} \bmod p$, and $a \equiv-x_{0}^{2} \bmod p$.

(D) $\operatorname{gcd}\left(a b x_{0}\left(b^{2}+4 a\right), p\right)=1$ and $x_{0}^{2}-x_{0} b-a \equiv 0 \bmod p$.

(E) $p \neq 2, \operatorname{gcd}\left(a b\left(b^{2}+4 a\right), p\right)=1, x_{0} \equiv b / 2 \bmod p$, and the order $\mathfrak{o}\left(m_{f}\right)$ of the polynomial $m_{f}(x)=x^{2}+\left(b^{2} / a+2\right) x+1$ in $\mathrm{GF}(p)[x]$ is odd.

(F) $\operatorname{gcd}\left(a b x_{0}\left(b^{2}+4 a\right) f\left(x_{0}\right), p\right)=1, x_{0} \not \equiv b / 2 \bmod p$ whenever $p \neq 2$, and the order $\mathfrak{o}\left(M_{f}\right)$ of $M_{f}(x)=x^{2}-\left(2+\left(b^{2}+4 a\right) / f\left(x_{0}\right)\right) x+1$ in $\operatorname{GF}(p)[x]$ does not divide $\mathfrak{o}\left(m_{f}\right)$. 
Proof. As we have mentioned, $a, b$, and $x_{0}$ can be used to define an infinite sequence by the recursion (2) if and only if the sequence $X\left(x_{0} ; a, b\right)$ with modulus $p$ defined by the recursion (1) does not contain 0 . From Lemma 1, the last statement holds if and only if $X\left(x_{0} ; a, b\right)$ with modulus $p$ is one of cases $(\mathrm{A}),(\mathrm{C}),(\mathrm{D}),(\mathrm{E})(1)$, and $(\mathrm{F})(1),(3)$, and (5). In fact, with modulus $p$, Lemma $1(\mathrm{~A})$ is case $(\mathrm{A})$, both Lemma $1(\mathrm{C})$ and $(\mathrm{D})$ together are case $(\mathrm{B})$, Lemma $1(\mathrm{E})(1)$ is case $(\mathrm{C})$, Lemma $1(\mathrm{~F})(1)$ is case $(\mathrm{D})$, Lemma $1(\mathrm{~F})(3)$ is case $(\mathrm{E})$, and Lemma $1(\mathrm{~F})(5)$ is case $(\mathrm{F})$.

The following two lemmas were obtained by Eichenauer-Herrmann and Topuzoğlu [7]. They are useful in describing the period length of the sequence $X\left(x_{0} ; a, b\right)$ with modulus $p^{k}$ for $k \geq 2$.

Lemma 3 ([7], Lemma 6). Let $p$ be a prime and let $k, a, b$, and $x_{0}$ be integers with $k \geq 2$ and $\operatorname{gcd}(a, p)=1$. Suppose that the sequence $X\left(x_{0} ; a, b\right)$ can be defined by the recursion (2). Let $\lambda_{k-1}$ and $\lambda_{k}$ be the period length of $X\left(x_{0} ; a, b\right)$ with modulus $p^{k-1}$ and $p^{k}$, respectively. Then

(A) $\lambda_{k}=\lambda_{k-1}$ for $x_{\lambda_{k-1}} \equiv x_{0} \bmod p^{k}$.

(B) $\lambda_{k}=\mathfrak{o}\left(-a x_{0}^{-2}\right) \lambda_{k-1}$ for $x_{\lambda_{k-1}} \not \equiv x_{0} \bmod p^{k}, \lambda_{k-1}=1$ and $\operatorname{gcd}(a+$ $\left.x_{0}^{2}, p\right)=1$, where $\mathfrak{o}\left(-a x_{0}^{-2}\right)$ is the multiplicative order of $-a x_{0}^{-2}$ in $\operatorname{GF}(p)$.

(C) $\lambda_{k}=p \lambda_{k-1}$ for $x_{\lambda_{k-1}} \not \equiv x_{0} \bmod p^{k}$, and either $\lambda_{k-1} \geq 2$ or $\lambda_{k-1}=1$ and $a \equiv-x_{0}^{2} \bmod p$.

The following lemma is a little bit different from the original lemmas in [7].

Lemma 4 ([7], Lemmas 7-9). Let $p$ be a prime and let $k, a, b$ and $x_{0}$ be integers with $k \geq 2$ and $\operatorname{gcd}(a, p)=1$. Suppose that the sequence $X\left(x_{0} ; a, b\right)$ can be defined by the recursion (2). Let $\lambda_{k-1}$ and $\lambda_{k}$ be the period lengths of $X\left(x_{0} ; a, b\right)$ with modulus $p^{k-1}$ and $p^{k}$, respectively.

(A) If $k \geq 3$ and $x_{\lambda_{k-1}} \not \equiv x_{0} \bmod p^{k}$, then $x_{\lambda_{k}} \not \equiv x_{0} \bmod p^{k+1}$.

(B) If $\lambda_{1} \geq 2$ and $x_{\lambda_{1}} \not \equiv x_{0} \bmod p^{2}$, then $x_{\lambda_{2}} \not \equiv x_{0} \bmod p^{3}$.

(C) If $\lambda_{1}=1, a \equiv-x_{0}^{2} \bmod p, x_{1} \not \equiv x_{0} \bmod p^{2}$ and $p \geq 5$, then $x_{\lambda_{2}} \not \equiv$ $x_{0} \bmod p^{3}$.

Proof. (B) and (C) are the same as Lemmas 8 and 9, respectively, in [7]. So, we prove (A) only. We follow the proof of Lemma 7 in [7] until we get the congruential equation

$$
x_{\mu \lambda_{k-1}} \equiv x_{0}+\mu\left(\alpha p^{k-1}+\beta p^{k}\right)+\left(\sum_{1 \leq j \leq \mu-1} j\right) \gamma \alpha p^{k} \bmod p^{k+1},
$$

where $\mu$ is any positive integer, $\alpha, \beta$, and $\gamma$ are some fixed integers with $\operatorname{gcd}(\alpha, p)=1$ and $\gamma=0$ if $p=2$. If the conditions of Lemma 3(B) are 
satisfied, we take $\mu=\mathfrak{o}\left(-a x_{0}^{-2}\right)$ and then the equation (4) becomes

$$
\begin{aligned}
x_{\lambda_{k}} & \equiv x_{0}+\mathfrak{o}\left(-a x_{0}^{-2}\right) \alpha p^{k-1}+\left(\mathfrak{o}\left(-a x_{0}^{-2}\right) \beta+\left(\sum_{1 \leq j \leq \mu-1} j\right) \gamma \alpha\right) p^{k} \\
& \not \equiv x_{0} \bmod p^{k+1}
\end{aligned}
$$

since $\operatorname{gcd}\left(\mathfrak{o}\left(-a x_{0}^{-2}\right) \alpha, p\right)=1$. If the conditions of Lemma $3(\mathrm{C})$ are satisfied, we take $\mu=p$ and then the equation (4) becomes $x_{\lambda_{k}} \equiv x_{0}+\alpha p^{k} \not \equiv$ $x_{0} \bmod p^{k+1}$ since $\sum_{1 \leq j \leq p-1} j \equiv 0 \bmod p$ if $p \geq 3$, and $\gamma=0$ if $p=2$. This completes the proof.

We are now ready to prove our main theorem of this section, which will describe all possible period lengths of the inversive congruential recursion with modulus $p^{k}$.

THEOREM 5. Let $p$ be a prime and let $k, a, b$ and $x_{0}$ be integers with $k \geq 1$. Suppose that the sequence $X\left(x_{0} ; a, b\right)$ with modulus $p^{k}$ can be defined by the recursion (2). Let $f(x)=x^{2}-b x-a$.

(A) If $a \equiv 0 \bmod p$ and $\operatorname{gcd}\left(b x_{0}, p\right)=1$, then the period length $L\left(x_{0} ; a, b ; p^{k}\right)=1$.

(B) Let $\operatorname{gcd}\left(a x_{0}, p\right)=1, a \equiv x_{0}^{2} \bmod p$, and $b \equiv 0 \bmod p$. Write $b=d p^{j}$ with $\operatorname{gcd}(d, p)=1$ whenever $b \neq 0$. Also write $f\left(x_{0}\right)=c p^{e}$ with $\operatorname{gcd}(c, p)=1$ when $f\left(x_{0}\right) \neq 0$.

(1) If either $f\left(x_{0}\right)=0$ or $k \leq e$, then $L\left(x_{0} ; a, b ; p^{k}\right)=1$.

(2) If $k=e+1$, then $L\left(x_{0} ; a, b ; p^{k}\right)=2$.

(3) If $e+1<k$ and either $b=0$ or $k \leq j$, then $L\left(x_{0} ; a, b ; p^{k}\right)=2$.

(4) If $k>e+1$ and $k>j$, then $L\left(x_{0} ; a, b ; p^{k}\right)=2 p^{k-\max \{j, e+1\}}$.

(C) Let $\operatorname{gcd}\left(a x_{0}\left(a-x_{0}^{2}\right), p\right)=1$ and $b \equiv 0 \bmod p$.

(1) If $b=0$, then $L\left(x_{0} ; a, b ; p^{k}\right)=2$.

(2) If $b=d p^{j}$ with $\operatorname{gcd}(d, p)=1$, then $L\left(x_{0} ; a, b ; p^{k}\right)=2$ if $1 \leq k \leq j$, and $L\left(x_{0} ; a, b ; p^{k}\right)=2 p^{k-j}$ if $k>j$.

(D) Let $\operatorname{gcd}(a b, p)=1, b \equiv 2 x_{0} \bmod p$, and $a \equiv-x_{0}^{2} \bmod p$. If $f\left(x_{0}\right) \neq 0$, write $f\left(x_{0}\right)=c p^{e}$ with $\operatorname{gcd}(c, p)=1$.

(1) If either $f\left(x_{0}\right)=0$ or $e \geq 2$ and $k \leq e$, then $L\left(x_{0} ; a, b ; p^{k}\right)=1$.

(2) If $k>e \geq 2$, then $L\left(x_{0} ; a, b ; p^{k}\right)=p^{k-e}$.

(3) If $p \geq 5$ and $e=1$, then $L\left(x_{0} ; a, b ; p^{k}\right)=p^{k-1}$.

(4) Let $p=3$ and $e=1$. Write $a+b^{2}=h 3^{s}$ for some integer $h$ with $\operatorname{gcd}(h, 3)=1$ whenever $a+b^{2} \neq 0$. Then $L\left(x_{0} ; a, b ; 3^{k}\right)=3$ if either $a+b^{2}=0$ or $2 \leq k \leq s$, and $L\left(x_{0} ; a, b ; 3^{k}\right)=3^{k-s+1}$ if $k \geq s+1$.

(E) Let $\operatorname{gcd}\left(a b x_{0}\left(b^{2}+4 a\right), p\right)=1$. Write $f\left(x_{0}\right)=c p^{e}$ with $\operatorname{gcd}(c, p)=1$ whenever $f\left(x_{0}\right) \neq 0$.

(1) If either $f\left(x_{0}\right)=0$ or $k \leq e$, then $L\left(x_{0} ; a, b ; p^{k}\right)=1$. 
(2) If $k>e \geq 2$, then $L\left(x_{0} ; a, b ; p^{k}\right)=\mathfrak{o}\left(-a x_{0}^{-2}\right) p^{k-e-1}$.

(3) Let $k>e=1$ and write $\mu=\mathfrak{o}\left(-a x_{0}^{-2}\right)$. Then $L\left(x_{0} ; a, b ; p^{k}\right)=$ $\mu$ if $x_{\mu} \equiv x_{0} \bmod p^{k}$, and $L\left(x_{0} ; a, b ; p^{k}\right)=\mu p^{k-t+1}$ if $t$ is the smallest integer satisfying $x_{\mu} \not \equiv x_{0} \bmod p^{t}$ and $3 \leq t \leq k$.

(F) Let $\operatorname{gcd}\left(a b x_{0}\left(b^{2}+4 a\right) f\left(x_{0}\right), p\right)=1$. Suppose that either $p>2, x_{0} \equiv$ $b / 2 \bmod p$ and the order $\lambda=\mathfrak{o}\left(m_{f}\right)$ of $m_{f}(x)=x^{2}+\left(b^{2} / a+2\right) x+1$ in $\mathrm{GF}(p)[x]$ is odd or the order $\mathfrak{o}\left(M_{f}\right)$ of the polynomial $M_{f}(x)=x^{2}-(2+$ $\left.\left(b^{2}+4 a\right) / f\left(x_{0}\right)\right) x+1$ in $\operatorname{GF}(p)[x]$ does not divide $\lambda$. Then $p$ is odd and $L\left(x_{0} ; a, b ; p^{k}\right)=\lambda$ if $x_{\lambda} \equiv x_{0} \bmod p^{k}$, and $L\left(x_{0} ; a, b ; p^{k}\right)=\lambda p^{k-t+1}$ if $t$ is the smallest positive integer satisfying $x_{\lambda} \not \equiv x_{0} \bmod p^{t}$ and $2 \leq t \leq k$.

Proof. Since $a, b$ and $x_{0}$ can be used to define the infinite sequence $X\left(x_{0} ; a, b\right)$ with modulus $p^{k}$ by the recursion $(2)$, we are going to prove this theorem according to all cases in Lemma 2.

(A) It is trivial for the case $a=0$. So, consider $a \neq 0$ and write $a=r p^{e}$ with $\operatorname{gcd}(r, p)=1$. Let $s$ be the nonnegative integer satisfying $e s<k \leq$ $e(s+1)$. We are going to prove this case by induction on $s$.

Since $x_{n+1} \equiv p^{e} r x_{n}^{-1}+b \bmod p^{t}$, we have $L\left(x_{0} ; a, b ; p^{t}\right)=1$ whenever $1 \leq t \leq e$. Suppose that for fixed integer $0 \leq s, L\left(x_{0} ; a, b ; p^{t}\right)=1$ for each $e s<t \leq e(s+1)$, or equivalently, there exists a positive integer $w_{s}$ so that for any es $<t \leq e(s+1), x_{n} \equiv u \bmod p^{t}$ is a constant for all $n \geq w_{s}$.

Now consider $e(s+1)<k \leq e(s+2)$. For any $n \geq w_{s}+1, x_{n} \equiv p^{e} r x_{n-1}^{-1}+$ $b \bmod p^{k}$. Since $n-1 \geq w_{s}$, the term $x_{n-1} \equiv u \bmod p^{k-e}$ is a constant. So, $p^{e} r x_{n-1}^{-1} \equiv p^{e} r u^{-1} \bmod p^{k}$ is a constant. Therefore, $x_{n} \equiv p^{e} r x_{n-1}^{-1}+b \bmod p^{k}$ is a constant for all $n \geq w_{s}+1$. Hence, $L\left(x_{0} ; a, b ; p^{k}\right)=1$.

From now on, we consider $\operatorname{gcd}(a, p)=1$. So, $X\left(x_{0} ; a, b\right)$ with modulus $p^{k}$ is purely periodic.

(B) From the definition, $x_{1} \equiv x_{0} \bmod p^{k}$ if and only if $f\left(x_{0}\right)=x_{0}^{2}-$ $b x_{0}-a \equiv 0 \bmod p^{k}$. So, if $f\left(x_{0}\right)=0$, then $L\left(x_{0} ; a, b ; p^{k}\right)=1$. Now, we consider $f\left(x_{0}\right) \neq 0$.

If $k \leq e$, it is trivial that $L\left(x_{0} ; a, b ; p^{k}\right)=1$. So, suppose $k>e$. Then $x_{1} \not \equiv$ $x_{0} \bmod p^{k}$. If $k=e+1$ and $p$ is odd, then, by Lemma $3(\mathrm{~B}), L\left(x_{0} ; a, b ; p^{e+1}\right)=$ 2 since $\mathfrak{o}\left(-a x_{0}^{-2}\right)=2$. From Lemma $3(\mathrm{C})$, if $p$ is even and $k=e+1$, then $L\left(x_{0} ; a, b ; p^{e+1}\right)=p=2$.

Now, suppose $k>e+1$. By the definition, $x_{0} \equiv x_{2} \bmod p^{k}$ if and only if $b^{2} x_{0}+a b+a x_{0} \equiv x_{0}\left(b x_{0}+a\right) \bmod p^{k}$. Simplifying the last congruential equation, $x_{0} \equiv x_{2} \bmod p^{k}$ if and only if $a b \equiv 0 \bmod p^{k}$. So, if either $b=0$ or $k \leq j$, then $L\left(x_{0} ; a, b ; p^{k}\right)=2$ by Lemma $3(\mathrm{~A})$. If $k>j$, then $L\left(x_{0} ; a, b ; p^{k}\right)=2 p^{k-\max \{j, m e+1\}}$ from Lemmas $3(\mathrm{C})$ and $4(\mathrm{~A})$.

(C) From Lemma $1(\mathrm{D}), L\left(x_{0} ; a, b ; p\right)=2$. By the definition, $x_{2} \equiv$ $a\left(a x_{0}^{-1}+b\right)^{-1}+b \bmod p^{k}$. If $b=0$, it is trivial that $L\left(x_{0} ; a, b ; p^{k}\right)=2$. Now suppose $b \neq 0$. After simplification, $x_{2} \equiv x_{0} \bmod p^{k}$ if and only if 
$\left(a-x_{0}^{2}\right) b \equiv-x_{0} b^{2} \bmod p^{k}$. The last congruential equation holds if and only if $1 \leq k \leq j$ since $b=d p^{j}$ and $\operatorname{gcd}\left(a-x_{0}^{2}, p\right)=1$. So, $L\left(x_{0} ; a, b ; p^{k}\right)=2$ if $1 \leq k \leq j$. Since $\left(a-x_{0}^{2}\right) b \not \equiv-x_{0} b^{2} \bmod p^{j+1}, L\left(x_{0} ; a, b ; p^{j+1}\right)=2 p$ by Lemma $3(\mathrm{C})$. If $k>j$, then $L\left(x_{0} ; a, b ; p^{k}\right)=2 p^{k-j}$ by Lemmas $3(\mathrm{C})$ and $4(\mathrm{~A})$ and $(\mathrm{B})$.

(D) By Lemma $1(\mathrm{E})(1), L\left(x_{0} ; a, b ; p\right)=1$. From the definition, $x_{1} \equiv$ $x_{0} \bmod p^{k}$ if and only if $f\left(x_{0}\right)=x_{0}^{2}-b x_{0}-a \equiv 0 \bmod p^{k}$. So, if either $f\left(x_{0}\right)=0$ or $k \leq e$, then $L\left(x_{0} ; a, b ; p^{k}\right)=1$. Suppose $f\left(x_{0}\right) \neq 0$ and $k>e$. Since $a \equiv-x_{0}^{2} \bmod p, L\left(x_{0} ; a, b ; p^{e+1}\right)=p$ by Lemma $3(\mathrm{C})$. If $e \geq 2$, then $L\left(x_{0} ; a, b ; p^{k}\right)=p^{k-e}$ by Lemmas $3(\mathrm{C})$ and $4(\mathrm{~A})$.

Suppose now $e=1$. From Lemmas $3(\mathrm{C})$ and $4(\mathrm{~A})$ and (C), $L\left(x_{0} ; a, b ; p^{k}\right)$ $=p^{k-1}$ if $p \geq 5$. So, suppose $p=3$. It is trivial that $L\left(x_{0} ; a, b ; 9\right)=3$. So, let $k \geq 3$. By the definition and a short calculation, $x_{3} \equiv x_{0} \bmod 3^{k}$ if and only if $a f\left(x_{0}\right) \equiv-b^{2} f\left(x_{0}\right) \bmod 3^{k}$. The last congruential equality is equivalent to $a \equiv-b^{2} \bmod 3^{k-1}$ since $f\left(x_{0}\right)=3 c$ with $\operatorname{gcd}(c, 3)=1$. Since $b \equiv 2 x_{0} \bmod 3$ and $a \equiv-x_{0}^{2} \bmod 3$, we have $a+b^{2} \equiv 0 \bmod 3$. If $a+b^{2}=0$, then $L\left(x_{0} ; a, b ; 3^{k}\right)=3$ by Lemmas $3(\mathrm{~A})$. So, suppose $a+b^{2} \neq 0$. If $s=1$, then $L\left(x_{0} ; a, b ; 3^{k}\right)=3^{k-1}$ by Lemmas $3(\mathrm{C})$ and $4(\mathrm{~A})$. Suppose $s \geq 2$. If $k \leq s$, then $L\left(x_{0} ; a, b ; 3^{k}\right)=3$. If $k \geq s+1$, then $L\left(x_{0} ; a, b ; 3^{k}\right)=3^{k-s}$ by Lemmas $3(\mathrm{C})$ and $4(\mathrm{~A})$.

(E) From the definition, $x_{0} \equiv x_{1} \bmod p^{k}$ if and only if $f\left(x_{0}\right) \equiv 0 \bmod p^{k}$. If either $f\left(x_{0}\right)=0$ or $k \leq e$, then $L\left(x_{0} ; a, b ; p^{k}\right)=1$. If $k>e \geq 2$, then $L\left(x_{0} ; a, b ; p^{k}\right)=\mathfrak{o}\left(-a x_{0}^{-2}\right) p^{k-e-1}$ from Lemmas 3(B), (C) and 4(A). Now suppose $e=1$. By Lemma 3(B) again, $L\left(x_{0} ; a, b ; p^{2}\right)=\lambda=\mathfrak{o}\left(-a x_{0}^{-2}\right)$. If $k>2$ and $x_{\lambda} \equiv x_{0} \bmod p^{k}$, then $L\left(x_{0} ; a, b ; p^{k}\right)=\lambda$. If $k>2$ and if $3 \leq t \leq k$ is the smallest positive integer satisfying $x_{\lambda} \not \equiv x_{0} \bmod p^{t}$, then $L\left(x_{0} ; a, b ; p^{k}\right)=\mathfrak{o}\left(-a x_{0}^{-2}\right) p^{k-t+1}$ by Lemmas $3(\mathrm{~B}),(\mathrm{C})$ and $4(\mathrm{~A})$.

(F) Under the assumption $\operatorname{gcd}(a b, p)=1$, the only case for $p=2$ is that $a \equiv 1 \equiv b \bmod 2$. In this case, $\mathfrak{o}\left(m_{f}\right)$ is 3 and so $L\left(x_{0} ; a, b ; 2\right)=$ 2. This implies that the sequence $X\left(x_{0} ; a, b\right)$ with modulus 2 contains 0 ; a contradiction. So, $p$ is odd.

From Lemma $1(\mathrm{~F})(3)$ and (5), $L\left(x_{0} ; a, b ; p\right)=\lambda=\mathfrak{o}\left(m_{f}\right)$. If $x_{\lambda} \equiv x_{0}$ $\bmod p^{k}$, then $L\left(x_{0} ; a, b ; p^{k}\right)=\mathfrak{o}\left(m_{f}\right)$ by Lemma $3(\mathrm{~A})$. If $2 \leq t \leq k$ is the smallest integer satisfying $x_{\lambda} \not \equiv x_{0} \bmod p^{e}$, then $L\left(x_{0} ; a, b ; p^{k}\right)=$ $\mathfrak{o}\left(m_{f}\right) p^{k-t+1}$ by Lemmas $3(\mathrm{~A}),(\mathrm{C}), 4(\mathrm{~A})$ and $(\mathrm{B})$. This completes the proof of this theorem.

The case Theorem 5(B)(3) with $j=1=e$ is consistent with the result obtained by Eichenauer, Lehn, and Topuzoğlu [4]. Also, cases (D)(4), (D)(5) with $s=1$, and (F) in Theorem 5 are consistent with results obtained by Eichenauer-Herrmann and Topuzoğlu [7]. Also, we have given conditions $x_{\lambda} \equiv x_{0}$ and $x_{\lambda} \not \equiv x_{0}$ modulo a prime power in both cases Theorem $5(\mathrm{E})(3)$ 
and $(\mathrm{F})$, respectively. We are going to modify these two conditions. First, we need the following

Lemma 6. Let $p$ be a prime and let $k, a, b$, and $x_{0}$ be integers with $k \geq 2$. Suppose that the sequence $X\left(x_{0} ; a, b\right): x_{0}, x_{1}, x_{2}, \ldots$ with modulus $p^{k}$ can be defined by the recursion (2). Let $U\left(1, x_{0} ; a, b\right): u_{0}, u_{1}, u_{2}, \ldots$ be the linear recurrence sequence of integers defined by $u_{0}=1, u_{1}=x_{0}$, and $u_{n+2}=$ $b u_{n+1}+a u_{n}$ for all $n \geq 0$. Then $\operatorname{gcd}\left(u_{n}, p\right)=1$ and $x_{n} \equiv u_{n+1} / u_{n} \bmod p^{k}$ for all $n \geq 0$.

Proof. From the definition, $x_{n}$ is not congruent to 0 modulo $p$ for all $n \geq 0$. So, $\operatorname{gcd}\left(u_{n}, p\right)=1$ by Lemma 1 . Since $x_{0} \equiv u_{1} \equiv u_{1} / u_{0} \bmod p^{k}$ and $u_{n+2} \equiv b u_{n+1}+a u_{n} \bmod p^{k}$, the result $x_{n} \equiv u_{n+1} / u_{n} \bmod p^{k}$ can be proved by induction on $n$.

The following theorem is a modification of the case Theorem $5(\mathrm{E})(3)$.

THEOREM 7. Let $p$ be an odd prime and let $k, a, b$ and $x_{0}$ be integers so that $k \geq 2, \operatorname{gcd}\left(a b x_{0}\left(b^{2}+4 a\right), p\right)=1$ and $x_{0}^{2}-b x_{0}-a=c p$ for some integer $c$ with $\operatorname{gcd}(c, p)=1$. Write $\left(a x_{0}^{-1}\right)^{p-1}-x_{0}^{p-1} \equiv v p \bmod p^{2}$ for some integer $v$, where $x_{0}^{-1}$ is the multiplicative inverse of $x_{0}$ modulo $p^{2}$.

(A) If $\operatorname{gcd}\left(c+2^{-1} v\left(x_{0}^{2}+a\right), p\right)=1$, then $L\left(x_{0} ; a, b ; p^{k}\right)=\mathfrak{o}\left(-a x_{0}^{-2}\right) p^{k-2}$, where $2^{-1}$ is the multiplicative inverse of 2 modulo $p$.

(B) If $c \equiv-v\left(x_{0}^{2}+a\right) / 2 \bmod p$, then $L\left(x_{0} ; a, b ; p^{3}\right)=\mathfrak{o}\left(-a x_{0}^{-2}\right)$ and, whenever $k \geq 4$, there is exactly one integer $0 \leq d<p^{k-3}$ so that $L\left(x_{0} ; a, b+\right.$ $\left.d p^{2} ; p^{k}\right)=\mathfrak{o}\left(-a x_{0}^{-2}\right)$.

Proof. We already know that $L\left(x_{0} ; a, b ; p\right)=1$ and $L\left(x_{0} ; a, b ; p^{2}\right)=$ $\mathfrak{o}\left(-a x_{0}^{-2}\right)=\lambda$ by Theorem $5(\mathrm{E})(3)$. Now, we consider the sequence $X\left(x_{0} ; a, b\right)$ with modulus $p^{3}$.

Since $\operatorname{gcd}\left(a x_{0}, p\right)=1$, there is an integer $\beta$ so that $\beta x_{0} \equiv-a \bmod p^{3}$. Write $x_{0}=\alpha$. Let $b_{c}=\beta+\alpha$ and $g(x)=x^{2}-b_{c} x-a$. So, $g\left(x_{0}\right) \equiv 0 \bmod p^{3}$. Moreover, $b-b_{c} \equiv x_{0}^{-1} c p$ mod $p^{3}$ since $f\left(x_{0}\right)=x_{0}^{2}-b x_{0}-a=c p$. Consider two corresponding linear recurrence sequences $U\left(1, x_{0} ; a, b\right): u_{0}, u_{1}, \ldots$ and $U\left(1, x_{0} ; a, b_{c}\right): u_{c, 0}, u_{c, 1}, \ldots$ defined as in Lemma 6 , respectively. By Lemma $6, x_{n} \equiv u_{n+1} / u_{n} \bmod p^{3}$ for all $n \geq 0$. Furthermore, it can be shown by induction on $n$ that $u_{c, n} \equiv \alpha^{n} \bmod p^{3}$ for all $n \geq 0$. Now, let $W\left(0,1 ; a, b_{c}\right): \omega_{c, 0}, \omega_{c, 1}, \ldots$ be the linear recurrence sequence defined by $\omega_{c, 0}=0, \omega_{c, 1}=1$, and $\omega_{c, n+2}=b_{c} \omega_{c, n+1}+a \omega_{c, n}$ for all $n \geq 0$. Since $\alpha \not \equiv \beta \bmod p$ from $\operatorname{gcd}\left(b^{2}+4 a, p\right)=1$, one can show by induction on $n$ that $\omega_{c, n} \equiv\left(\alpha^{n}-\beta^{n}\right) /(\alpha-\beta) \bmod p^{3}$ for all $n \geq 0$.

It is easy to see from the definition that $u_{0} \equiv u_{c, 0}, u_{1} \equiv u_{c, 1}, u_{2} \equiv u_{c, 2}+$ $\omega_{c, 1} u_{c, 1} x_{0}^{-1} c p$ and $u_{3} \equiv u_{c, 3}+\left(\omega_{c, 1} u_{c, 2}+\omega_{c, 2} u_{c, 1}\right) x_{0}^{-1} c p+\omega_{c, 1} u_{c, 1}\left(x_{0}^{-1} c p\right)^{2}$ $\bmod p^{3}$. Let $\sigma_{n}=\sum_{1 \leq j \leq n-1} \omega_{c, j} u_{c, n-j}$ and $\tau_{n}=\sum_{1 \leq j \leq n-2} \omega_{c, j} \sigma_{n-j}$ for all $n \geq 3$, and let $\sigma_{2} \stackrel{=}{=} \omega_{c, 1} u_{c, 1}$. One can show by induction on $n$ that 
$u_{n} \equiv u_{c, n}+\sigma_{n} x_{0}^{-1} c p+\tau_{n}\left(x_{0}^{-1} c p\right)^{2} \bmod p^{3}$ for all $n \geq 3$. Since $\omega_{c, n} \equiv$ $\left(\alpha^{n}-\beta^{n}\right) /(\alpha-\beta)$ and $u_{c, n} \equiv \alpha^{n} \bmod p^{3}$, we have, after a short computation,

$$
\begin{aligned}
\sigma_{n} & =\sum_{1 \leq j \leq n-1} \omega_{c, j} u_{c, n-j} \equiv\left(\sum_{0 \leq j \leq n-1} \alpha^{n-j}\left(\alpha^{j}-\beta^{j}\right)\right) /(\alpha-\beta) \\
& \equiv n \alpha^{n} /(\alpha-\beta)-\alpha\left(\alpha^{n}-\beta^{n}\right) /(\alpha-\beta)^{2} \bmod p^{3}
\end{aligned}
$$

and

(6)

$$
\begin{aligned}
\tau_{n}= & \sum_{1 \leq j \leq n-2} \omega_{c, j} \sigma_{n-j} \\
\equiv & (\alpha-\beta)^{-2}\left(\sum_{0 \leq j \leq n-2}\left(\alpha^{j}-\beta^{j}\right)(n-j) \alpha^{n-j}\right) \\
& -\alpha(\alpha-\beta)^{-3}\left(\sum_{0 \leq j \leq n-2}\left(\alpha^{j}-\beta^{j}\right)\left(\alpha^{n-j}-\beta^{n-j}\right)\right) \\
\equiv & (\alpha-\beta)^{-2}\left((n+2)(n-1) \alpha^{n} / 2-n \alpha^{2}\left(\alpha^{n-1}-\beta^{n-1}\right) /(\alpha-\beta)\right. \\
& \left.+\left((n-2) \alpha^{2} \beta^{n}-(n-1) \alpha^{3} \beta^{n-1}+\alpha^{n+1} \beta\right) /(\alpha-\beta)^{2}\right) \\
& -\alpha(\alpha-\beta)^{-3}\left((n-1) \alpha^{n}-\beta^{2}\left(\alpha^{n-1}-\beta^{n-1}\right) /(\alpha-\beta)\right. \\
& \left.-\alpha^{2}\left(\alpha^{n-1}-\beta^{n-1}\right) /(\alpha-\beta)+(n-1) \beta^{n}\right) \bmod p^{3} .
\end{aligned}
$$

Since $\beta x_{0} \equiv-a \bmod p^{3}$, we have $-a x_{0}^{-2} \equiv \alpha \beta^{-1} \bmod p$. This implies $\mathfrak{o}\left(-a x_{0}^{-2}\right)=\mathfrak{o}\left(m_{f}\right)=\lambda$. So, $\beta^{\lambda} \equiv \alpha^{\lambda} \bmod p$. Hence, $\alpha^{p-1} \equiv \beta^{p-1} \bmod p$. Write $\beta^{p-1} \equiv \alpha^{p-1}+v p \bmod p^{2}$ for some integer $v$. Note that $x_{0} \equiv x_{\lambda}$ $\bmod p^{3}$ if and only if $x_{0} \equiv x_{p-1} \bmod p^{3}$ by Lemma $3(\mathrm{C})$. So, we consider $x_{p-1}$ instead of $x_{\lambda}$. By formula (5) and (6) and simplification,

$$
\begin{aligned}
& \left(x_{0}^{-1} c p\right) \sigma_{p-1} \\
& \equiv-\alpha^{p-1}(\alpha-\beta)^{-1}\left(x_{0}^{-1} c p\right)+\left((\alpha-\beta)^{-1}+\alpha v(\alpha-\beta)^{-2}\right) x_{0}^{-1} c p^{2} \bmod p^{3} \\
& \left(x_{0}^{-1} c p\right) \sigma_{p} \\
& \equiv-\alpha^{p}(\alpha-\beta)^{-1}\left(x_{0}^{-1} c p\right)+\left(\alpha(\alpha-\beta)^{-1}+\alpha \beta v(\alpha-\beta)^{-2}\right) x_{0}^{-1} c p^{2} \bmod p^{3} \\
& \left(x_{0}^{-1} c p\right)^{2} \tau_{p-1} \equiv 3 \alpha(\alpha-\beta)^{-3}\left(x_{0}^{-1} c p\right)^{2} \bmod p^{3}
\end{aligned}
$$

and

$$
\left(x_{0}^{-1} c p\right)^{2} \tau_{p} \equiv\left(\alpha^{2}+2 \alpha \beta\right)(\alpha-\beta)^{-3}\left(x_{0}^{-1} c p\right)^{2} \bmod p^{3} .
$$

By Lemma 6 and a short computation, we have

$$
\begin{aligned}
& x_{p-1} \equiv u_{p} / u_{p-1} \\
& \quad \equiv\left(u_{c, p}+\sigma_{p} x_{0}^{-1} c p+\sigma_{p}\left(x_{0}^{-1} c p\right)^{2}\right) /\left(u_{c, p-1}+\sigma_{p-1} x_{0}^{-1} c p+\sigma_{p-1}\left(x_{0}^{-1} c p\right)^{2}\right) \\
& \quad \equiv \alpha+\left(-v c(\alpha-\beta)^{-1}-2 c^{2} \alpha^{-1}(\alpha-\beta)^{-2}\right) p^{2} \bmod p^{3} .
\end{aligned}
$$

Since $x_{0}=\alpha, x_{p-1} \equiv x_{0} \bmod p^{3}$ if and only if $0 \equiv-v c(\alpha-\beta)^{-1}-$ $2 c^{2} \alpha^{-1}(\alpha-\beta)^{-2} \bmod p$. The last congruential equality is equivalent to 
$c \equiv-v \alpha(\alpha-\beta) / 2 \equiv-v\left(x_{0}^{2}+a\right) / 2 \bmod p$. By Lemmas $3(\mathrm{C})$ and $4(\mathrm{~A})$, if $\operatorname{gcd}\left(c+v\left(x_{0}^{2}+a\right), p\right)=1$, then $L\left(x_{0} ; a, b ; p^{3}\right)=\mathfrak{o}\left(-a x_{0}^{-2}\right) p$, and so $L\left(x_{0} ; a, b ; p^{k}\right)=\mathfrak{o}\left(-a x_{0}^{-2}\right) p^{k-2}$ for all $k \geq 3$.

Suppose $c \equiv-v\left(x_{0}^{2}+a\right) / 2 \bmod p$. Then $\operatorname{gcd}(v, p)=1$ since $\operatorname{gcd}\left(c\left(x_{0}^{2}+\right.\right.$ $a), p)=1$. Note that $L\left(x_{0} ; a, b ; p^{3}\right)=\mathfrak{o}\left(-a x_{0}^{-2}\right)$ from Lemma $3(\mathrm{~A})$. Assume $k>3$ and let $3 \leq t<k$ be any integer satisfying $L\left(x_{0} ; a, b ; p^{i}\right)=\mathfrak{o}\left(-a x_{0}^{-2}\right)$ for all $2 \leq i \leq t$. Write $x_{p-1} \equiv x_{0}+\xi p^{t} \bmod p^{t+1}$. Take any integer $0 \leq d<p$ and consider the sequence $X\left(x_{0} ; a, b+d p^{t-1}\right): x_{d, 0}, x_{d, 1}, \ldots$ with modulus $p^{t+1}$. Consider the corresponding linear recurrence sequence $U\left(1, x_{0} ; a, b+\right.$ $\left.d p^{t-1}\right): u_{d, 0}, u_{d, 1}, \ldots$ By Lemma $6, x_{d, n} \equiv u_{d, n+1} / u_{d, n} \bmod p^{t+1}$ for all $n \geq 0$. Let $W(0,1 ; a, b): w_{0}, w_{1}, \ldots$ be the linear recurrence sequence defined by $w_{0}=0, w_{1}=1$, and $w_{n+2}=b w_{n+1}+a w_{n}$ for all $n \geq 0$. By similar arguments, one can show by induction on $n$ that for all $n \geq 2$,

$$
u_{d, n} \equiv u_{n}+\sum_{1 \leq j \leq n-1} w_{j} u_{n-j} d p^{t-1} \bmod p^{t+1} .
$$

It is easy to show by induction on $n$ that $w_{1} \equiv \omega_{c, 1}$ and $w_{n} \equiv \omega_{c, n}+$ $\left(\sum_{1 \leq j \leq n-1} \omega_{c, j} \omega_{c, n-j}\right) x_{0}^{-1} c p \bmod p^{2}$ for all $n \geq 2$. Since $u_{1} \equiv u_{c, 1}$ and $u_{n} \equiv u_{c, n}+\sigma_{n} x_{0}^{-1} c p \bmod p^{2}$ for all $n \geq 2$, (7) becomes, for all $n \geq 3$,

$$
\begin{aligned}
u_{d, n} \equiv & u_{n}+\sum_{1 \leq j \leq n-1} \omega_{c, j} u_{n-j} d p^{t-1} \\
& +\sum_{2 \leq j \leq n-1} u_{n-j}\left(\sum_{1 \leq i \leq j-1} \omega_{c, i} \omega_{c, j-i}\right) x_{0}^{-1} c d p^{t} \\
\equiv & u_{n}+\sum_{1 \leq j \leq n-1} \omega_{c, j} u_{c, n-j} d p^{t-1}+\sum_{1 \leq j \leq n-2} \omega_{c, j} \sigma_{n-j} x_{0}^{-1} c d p^{t} \\
& +\sum_{2 \leq j \leq n-1} u_{n-j}\left(\sum_{1 \leq i \leq j-1} \omega_{c, i} \omega_{c, j-i}\right) x_{0}^{-1} c d p^{t} \bmod p^{t+1} .
\end{aligned}
$$

Let $\chi_{n}=\sum_{2 \leq j \leq n-1} u_{n-j}\left(\sum_{1 \leq i \leq j-1} \omega_{c, i} \omega_{c, n-i}\right)$ for all $n \geq 3$. From the definitions of $\sigma_{n}$ and $\tau_{n}$, (8) can be rewritten as

$$
u_{d, n} \equiv u_{n}+\sigma_{n} d p^{t-1}+\left(\tau_{n}+\chi_{n}\right) x_{0}^{-1} c d p^{t} \bmod p^{t+1} \quad \text { for all } n \geq 3 .
$$

Since $u_{n} \equiv u_{c, n} \bmod p$ for all $n \geq 0$, we have

$$
\begin{aligned}
\chi_{n}= & \sum_{2 \leq j \leq n-1} u_{n-j}\left(\sum_{1 \leq i \leq j-1} \omega_{c, i} \omega_{c, j-i}\right) \\
\equiv & (n+1)(n-2) \alpha^{n} 2^{-1}(\alpha-\beta)^{-2} \\
& +\left((n-1) \alpha \beta^{n+1}-n \alpha^{2} \beta^{n}+2 \alpha^{n} \beta^{2}-\alpha^{n-1} \beta^{3}\right)(\alpha-\beta)^{-4} \\
& -(n-2) \alpha^{n}(\alpha+\beta)(\alpha-\beta)^{-3} \\
& +\left(\alpha \beta^{n}-\alpha^{n-1} \beta^{2}\right)(\alpha+\beta)(\alpha-\beta)^{-4} \bmod p
\end{aligned}
$$


for all $n \geq 3$. Therefore,

$$
\begin{aligned}
u_{d, p-1} \equiv & u_{p-1}-\alpha^{p-1}(\alpha-\beta)^{-1} d p^{t-1}+\left((\alpha-\beta)^{-1}+\alpha v(\alpha-\beta)^{-2}\right) d p^{t} \\
& +\left(8 \alpha^{2}+4 \alpha \beta+2 \beta^{2}\right) \alpha^{-1}(\alpha-\beta)^{-3} x_{0}^{-1} c d p^{t} \bmod p^{t+1},
\end{aligned}
$$

and

$$
\begin{aligned}
u_{d, p} \equiv & u_{p}-\alpha^{p}(\alpha-\beta)^{-1} d p^{t-1}+\left(\alpha(\alpha-\beta)^{-1}+\alpha \beta v(\alpha-\beta)^{-2}\right) d p^{t} \\
& +\left(2 \alpha^{2}+6 \alpha \beta+2 \beta^{2}\right)(\alpha-\beta)^{-3} x_{0}^{-1} c d p^{t} \bmod p^{t+1} .
\end{aligned}
$$

Since $x_{p-1} \equiv x_{0}+\xi p^{t} \bmod p^{t+1}$ and $u_{p-1} \equiv \alpha^{p-1} \equiv 1, x_{0} \equiv \alpha$, and $c \equiv$ $-v \alpha(\alpha-\beta) / 2 \bmod p$, we have $x_{d, p-1} \equiv u_{d, p} / u_{d, p-1} \equiv x_{0}+\left(\xi+2 \alpha^{2} v(\alpha-\right.$ $\left.\beta)^{-2} d\right) p^{t} \bmod p^{t+1}$. This implies that $x_{d, p-1} \equiv x_{d, 0} \equiv x_{0} \bmod p^{t+1}$ if and only if $d \equiv-\xi(\alpha-\beta)^{2} /\left(2 \alpha^{2} v\right) \bmod p$. Since $(\alpha-\beta)^{2} \equiv b^{2}+4 a \bmod p$, $x_{d, p-1} \equiv x_{d, 0} \bmod p^{t+1}$ if and only if $d \equiv-\xi\left(b^{2}+4 a\right) /\left(2 x_{0}^{2} v\right) \bmod p$. We have shown that there is exactly one integer $0 \leq d<p$ so that $L\left(x_{0} ; a, b+\right.$ $\left.d p^{t-1} ; p^{t+1}\right)=\mathfrak{o}\left(-a x_{0}^{-2}\right)$ whenever $L\left(x_{0} ; a, b ; p^{t}\right)=\mathfrak{o}\left(-a x_{0}^{-2}\right)$. Case (B) of this theorem holds by taking such $d$ repeatedly starting from $t=2$. This completes the proof.

The following theorem modifies Theorem $5(\mathrm{~F})$.

THEOREM 8. Let $p>2$ and $\operatorname{gcd}\left(a b\left(b^{2}+4 a\right)\left(x_{0}^{2}-b x_{0}-a\right), p\right)=1$. Suppose that either $x_{0} \equiv b / 2 \bmod p$ and the order $\lambda=\mathfrak{o}\left(m_{f}\right)$ of $m_{f}(x)=$ $x^{2}+\left(b^{2} / a+2\right) x+1$ in $\mathrm{GF}(p)[x]$ is odd or $x_{0} \not \equiv b / 2 \bmod p$ and the order $\mathfrak{o}\left(M_{f}\right)$ of $M_{f}(x)=x^{2}-\left(2+\left(b^{2}+4 a\right) / f\left(x_{0}\right)\right) x+1$ in $\operatorname{GF}(p)[x]$ does not divide $\mathfrak{o}\left(m_{f}\right)$. Let $k \geq 2$.

(A) If $L\left(x_{0} ; a, b ; p^{k-1}\right)=\mathfrak{o}\left(m_{f}\right)$, then there exists exactly one integer $0 \leq d<p^{k-2}$ such that $L\left(x_{0} ; a, b+d p ; p^{k}\right)=\mathfrak{o}\left(m_{f}\right)$.

(B) If $x_{\lambda} \equiv x_{0}+v p \bmod p^{2}$ for some integer $0 \leq v<p$, then $L\left(x_{0} ; a, b+\right.$ $\left.d p ; p^{2}\right)=\mathfrak{o}\left(m_{f}\right)$ if and only if $d \equiv v\left(b^{2}+4 a\right) / 2 \lambda\left(x_{0}^{2}-b x_{0}-a\right) \bmod p$.

Proof. Let $d$ be any integer. As in the proof of the last theorem, consider the sequences $X\left(x_{0} ; a, b\right): x_{0}, x_{1}, \ldots$ and $X\left(x_{0} ; a, b+d p^{k-1}\right)$ : $x_{d, 0}, x_{d, 1}, \ldots$ with modulus $p^{k}$ and their corresponding linear recurrence sequences $U\left(1, x_{0} ; a, b\right): u_{0}, u_{1}, \ldots$ and $U\left(1, x_{0} ; a, b+d p^{k-1}\right): u_{d, 0}, u_{d, 1}, \ldots$, respectively. From Lemma $6, x_{n} \equiv u_{n+1} / u_{n}$ and $x_{d, n} \equiv u_{d, n+1} / u_{d, n} \bmod p^{k}$ for all $n \geq 0$. Moreover, let $W(0,1 ; a, b): w_{0}, w_{1}, w_{2}, \ldots$ be the same linear recurrence sequence as in the proof of Theorem 7 . One can show by induction on $n$ that for all $n \geq 2$,

$$
u_{d, n} \equiv u_{n}+\sum_{1 \leq j \leq n-1} w_{j} u_{n-j} d p^{k-1} \bmod p^{k} .
$$

Note that $f(x)=x^{2}-b x-a$ is the characteristic polynomial for both sequences $U\left(1, x_{0} ; a, b\right)$ and $W(0,1 ; a, b)$ with modulus $p$ (or equivalently, over $\operatorname{GF}(p))$. Since $\operatorname{gcd}\left(b^{2}+4 a, p\right)=1, f(x)$ is not a square in $\operatorname{GF}(p)[x]$. 
Let $\alpha, \beta \in \mathrm{GF}\left(p^{2}\right)$ be the roots of $f(x)$. It is easy to see that for all $n \geq 0$, $u_{n}=\left(\left(x_{0}-\beta\right) \alpha^{n}+\left(\alpha-x_{0}\right) \beta^{n}\right) /(\alpha-\beta)$ in $\operatorname{GF}\left(p^{2}\right)$. In particular, $u_{\lambda}=\alpha^{\lambda}$ in $\operatorname{GF}\left(p^{2}\right)$ where $\lambda=\mathfrak{o}\left(m_{f}\right)$. It can also be shown by induction on $n$ that for all $n \geq 0, w_{n}=\left(\alpha^{n}-\beta^{n}\right) /(\alpha-\beta)$ in $\operatorname{GF}\left(p^{2}\right)$. So, in $\operatorname{GF}\left(p^{2}\right)$,

$$
\begin{aligned}
\sum_{1 \leq j \leq n-1} w_{j} u_{n-j}= & \sum_{0 \leq j \leq n-1}\left(\left(x_{0}-\beta\right) \alpha^{n}+\left(\alpha-x_{0}\right) \beta^{n}\left(\alpha \beta^{-1}\right)^{j}\right. \\
& \left.-\left(x_{0}-\beta\right) \alpha^{n}\left(\alpha^{-1} \beta\right)^{j}-\left(\alpha-x_{0}\right) \beta^{n}\right)(\alpha-\beta)^{-2} \\
= & \left(n\left(x_{0}-\beta\right) \alpha^{n}+\left(\alpha-x_{0}\right) \beta\left(\alpha^{n}-\beta^{n}\right)(\alpha-\beta)^{-1}\right. \\
& \left.-\left(x_{0}-\beta\right) \alpha\left(\alpha^{n}-\beta^{n}\right)(\alpha-\beta)^{-1}-n\left(\alpha-x_{0}\right) \beta^{n}\right)(\alpha-\beta)^{-2}
\end{aligned}
$$

for all $n \geq 2$. In particular,

$$
\sum_{1 \leq j \leq \lambda-1} w_{j} u_{\lambda-j}=\lambda \alpha^{\lambda}\left(2 x_{0}-\beta-\alpha\right)(\alpha-\beta)^{-2}
$$

and

$$
\sum_{1 \leq j \leq \lambda} w_{j} u_{\lambda-j+1}=\lambda \alpha^{\lambda}\left(x_{0} \alpha+x_{0} \beta-2 \alpha \beta\right)(\alpha-\beta)^{-2} .
$$

Note that both values in (10) and (11) are in $\mathrm{GF}(p)$, and so can be viewed as an integer modulo $p$. Since $x_{\lambda} \equiv x_{0} \bmod p^{k-1}$, we can write $x_{\lambda} \equiv$ $x_{0}+v p^{k-1} \bmod p^{k}$ for some integer $0 \leq v<p$. So, $u_{\lambda+1} \equiv u_{\lambda} x_{0}+\alpha^{\lambda} v p^{k-1}$ $\bmod p^{k}$. Using this result and formulas (9)-(11), we have

$$
\begin{aligned}
x_{d, \lambda} & \equiv u_{d, \lambda+1} / u_{d, \lambda} \equiv x_{0}+\left(v-2 d \lambda\left(x_{0}^{2}-(\alpha+\beta) x_{0}+2 \alpha \beta\right)(\alpha-\beta)^{-2}\right) p^{k-1} \\
& \equiv x_{0}+\left(v-2 d \lambda\left(x_{0}^{2}-b x_{0}-a\right) /\left(b^{2}+4 a\right)\right) p^{k-1} \bmod p^{k} .
\end{aligned}
$$

Therefore, $x_{d, \lambda} \equiv x_{0} \bmod p^{k}$ if and only if $d \equiv v\left(b^{2}+4 a\right) /\left(2 \lambda\left(x_{0}^{2}-b x_{0}-\right.\right.$ a)) $\bmod p$. Since $\operatorname{gcd}\left(2 \lambda\left(x_{0}^{2}-b x_{0}-a\right), p\right)=1$, such a $d$ exists uniquely when we consider $0 \leq d<p$. This theorem is obtained by taking such $d$ repeatedly starting from $k=2$.

Note that the result of Theorem $8(\mathrm{~B})$ is consistent with the result obtained by Eichenauer-Herrmann [6]. The following result is an easy application of Theorem 5, which is consistent with results obtained by Huber [9]. We will use the usual notation $p^{t} \| m$ for $p^{t} \mid m$ but $p^{t+1} \nmid m$.

COROLlary 9. Let $m>1$ be a composite integer and let $a, b$ and $x_{0}$ be integers so that the infinite sequence $X\left(x_{0} ; a, b\right)$ with modulus $m$ can be defined by the recursion $(2)$. Then $X\left(x_{0} ; a, b\right)$ has the maximal period length among all inversive congruential pseudorandom number generators with modulus $m$ if and only if for any prime divisor of $m$ one of the following conditions holds:

(A) $2^{t} \| m, \operatorname{gcd}\left(a x_{0}, 2\right)=1$ and either $b \equiv 0 \bmod 2$ when $t=1$ or $a \equiv 1 \bmod 4, b \equiv 2 \bmod 4$, and $x_{0} \equiv 1 \bmod 2$ when $t \geq 2$. 
(B) $p^{t} \| m$ with $p$ odd, $\operatorname{gcd}\left(a b x_{0}\left(b^{2}+4 a\right)\left(x_{0}^{2}-b x_{0}-a\right), p\right)=1$, the order $\lambda=\mathfrak{o}\left(m_{f}\right)$ of the polynomial $m_{f}(x)=x^{2}+\left(b^{2} / a+2\right) x+1$ in $\mathrm{GF}(p)[x]$ equals $(p+1) / 2, x_{(p+1) / 2} \not \equiv x_{0} \bmod p^{2}$ whenever $t \geq 2$, and either $x_{0} \equiv b / 2 \bmod p$ and $p \equiv 1 \bmod 4$ or $x_{0} \not \equiv b / 2 \bmod p$ and the order $\mathfrak{o}\left(M_{f}\right)$ of $M_{f}(x)=$ $x^{2}-\left(2+\left(b^{2}+4 a\right) / f\left(x_{0}\right)\right) x+1$ in $\operatorname{GF}(p)[x]$ does not divide $(p+1) / 2$.

Proof. Note that the infinite sequence $X\left(x_{0} ; a, b\right)$ with modulus $m$ has the maximal period length among all inversive congruential pseudorandom number generators with modulus $m$ if and only if for any prime factor $p$ of $m, X\left(x_{0} ; a, b\right)$ with modulus $p^{t}$ has the maximal period length among all inversive congruential pseudorandom number generators with modulus $p^{t}$, where $p^{t} \| m$.

Let $2^{t} \| m$. Then $\operatorname{gcd}\left(x_{0}, 2\right)=1$. From Theorem 5 , the cases we have to consider are either $a \equiv 0$ or $b \equiv 0 \bmod 2$, but not both. If $t=1$, the sequence $X\left(x_{0} ; a, b\right)$ with modulus 2 having the maximal period length among all inversive congruential pseudorandom number generators with modulus 2 if and only if $\operatorname{gcd}\left(a x_{0}, 2\right)=1$ and $b \equiv 0 \bmod 2$. If $t \geq 2, X\left(x_{0} ; a, b\right)$ with modulus $2^{t}$ has the maximal period length among all inversive congruential pseudorandom number generators with modulus $2^{t}$ if and only if the case Theorem 5(B)(4) holds. This proves (A).

Let $p^{t} \| m$ with $p$ odd. Note that if either $L\left(x_{0} ; a, b ; p\right)=p-1$ or $L\left(x_{0} ; a, b ; p\right)=p+1$, then $X\left(x_{0} ; a, b\right)$ with modulus $p$ contains 0 . So, the sequence $X\left(x_{0} ; a, b\right)$ with modulus $p$ having the maximal period length among all inversive congruential pseudorandom number generators with modulus $p$ if and only if $L\left(x_{0} ; a, b ; p\right)=(p+1) / 2$, because $L\left(x_{0} ; a, b ; p\right)$ divides either $p-1$ or $p+1$ by Theorem 5 . The last statement holds if and only if it is the case Theorem $5(\mathrm{~F})$ together with $x_{\lambda} \not \equiv x_{0} \bmod p^{2}$ when $t \geq 2$. This completes the proof.

Let $m$ be a composite positive integer and let $m=p_{1}^{r_{1}} \ldots p_{t}^{r_{t}}$ be the prime factorization of $m$, where $p_{1}, \ldots, p_{t}$ are distinct primes and $r_{1}, \ldots, r_{t}$ are positive integers. To get a sequence with modulus $m$ having the maximal period length, we can first take a sequence $X\left(x_{i, 0} ; a_{i}, b_{i}\right)$ with each modulus $p_{i}^{r_{i}}, 1 \leq i \leq t$, satisfying conditions (A) or (B) of Corollary 9 , and then use the Chinese Remainder Theorem to get a sequence $X\left(x_{0} ; a, b\right)$ with modulus $m$. If $p=2$ is a prime divisor of $m$, it is easy to use the condition (A) of Corollary 9 to get the desired sequence with modulus a power of 2 . If $p$ is an odd prime factor of $m$, we have to do much more work.

Let $p$ be an odd prime and $k$ be a positive integer. To get a sequence $X\left(x_{0} ; a, b\right)$ with modulus $p^{k}$ which satisfies the condition (B) of Corollary 9 , we have first to find numbers $a$ and $b$ so that the order of the polynomial $m_{f}(x)=x^{2}+\left(b^{2} / a+2\right) x+1$ in $\operatorname{GF}(p)[x]$ is $(p+1) / 2$. We can pick up suitable $a$ and $b$ in the following way. 
Note that Chou [1] gave several methods to find polynomials over $\operatorname{GF}(p)$ of order $p+1$. We can first use his methods to find a polynomial $m(x)=x^{2}-$ $c x+1,0 \leq c<p$, of order $p+1$ in $\operatorname{GF}(p)[x]$. Consider the sequence $v_{0}, v_{1}, \ldots$ defined by $v_{0}=2, v_{1}=c$, and $v_{n+2}=c v_{n+1}-v_{n}$ for all $n \geq 0$. Then $v_{n}=\alpha^{n}+\alpha^{p n}$ for all $n \geq 0$, where $\alpha$ is a root of $m(x)$ in $\operatorname{GF}\left(p^{2}\right)$. So, $c^{2}-2=$ $v_{2}=\alpha^{2}+\alpha^{2 p}$ in $\operatorname{GF}\left(p^{2}\right)$. Since $m(x)$ is of order $p+1, m_{f}(x)=x^{2}-v_{2} x+1$ is of order $(p+1) / 2$ in $\operatorname{GF}(p)[x]$. From the relation $c \equiv-b^{2} / a-2 \bmod p$, we can get $p-1$ desired pairs of numbers $a$ and $b$ which are not congruent to $0 \bmod p$.

Once we have suitable numbers $a$ and $b$, we can choose a suitable number $x_{0}$ as follows. Note that the period length of the sequence $v_{0}, v_{1}, \ldots$ over $\operatorname{GF}(p)$ is $p+1$. Any polynomial $x^{2}-d x+1$ over $\mathrm{GF}(p)$ is of order $(p+1) / 2$ if and only if $d \equiv v_{2 n}$ for some positive integer $n$ satisfying $\operatorname{gcd}(n,(p+1) / 2)=1$. Take any integer $w$ so that $w \not \equiv v_{2 n} \bmod p$ for any integer $0 \leq n<(p+1) / 2$. Then the order of $M_{f}(x)=x^{2}-w x+1$ in $\mathrm{GF}(p)[x]$ does not divide $(p+1) / 2$. Let $t \equiv\left(b^{2}+4 a\right) /(w-2) \bmod p$. If the congruential equation $x^{2}-b x+a \equiv t \bmod p$ does not have a solution, we pick up another $w$ and then find a new $t$ and solve this new congruential equation. Suppose that the last congruential equation has a solution, say $x_{0}$. If $k=1$, the sequence $X\left(x_{0} ; a, b\right)$ is as required. If $k \geq 2$, we check the condition $x_{(p+1) / 2} \not \equiv x_{0} \bmod p^{2}$. If the condition is satisfied, we are done; otherwise, the sequences $X\left(x_{0}+c p ; a, b\right), 1 \leq c<p$, are as desired.

3. Generalized inversive congruential recursion. Let $p$ be a prime and $k$ be a positive integer again. In this section, we are going to study the sequence $X\left(x_{0} ; a, b\right)$ with modulus $p^{k}$ which is defined by the recursion (3). Let $L_{G}\left(x_{0} ; a, b ; p^{k}\right)$ be the period length of the sequence $X\left(x_{0} ; a, b\right)$ with modulus $p^{k}$ which is defined by the recursion (3). As we have mentioned in Section 1 , if $X\left(x_{0} ; a, b\right)$ with modulus $p$ does not contain 0 , then $L_{G}\left(x_{0} ; a, b ; p^{k}\right)=L\left(x_{0} ; a, b ; p^{k}\right)$. So, if $X\left(x_{0} ; a, b\right)$ with modulus $p$ does not contain 0 , then $L_{G}\left(x_{0} ; a, b ; p^{k}\right)$ must be one of the cases in Theorem 5 . Hence, we will concentrate on the case where $X\left(x_{0} ; a, b\right)$ with modulus $p$ contains 0 . We need the following lemma.

LEMMA 10. Let $p$ be a prime and $k$ be a positive integer so that either $k \geq 1$ if $p$ is odd or $k \geq 3$ if $p=2$. Let $a, b$ and $x_{0}$ be integers and let the sequence $X\left(x_{0} ; a, b\right): x_{0}, x_{1}, \ldots$ with modulus $p^{k}$ be defined by the recursion (3). If there is a nonnegative integer $t$ so that $x_{t} \equiv 0 \bmod p$, then $x_{t+1} \equiv b \bmod p^{k}$.

Proof. Write $\mu=\phi\left(p^{k}\right)=(p-1) p^{k-1}$ and $x_{t} \equiv r p \bmod p^{k}$ for some integer $r$. Then we have $x_{t+1} \equiv a(r p)^{\mu-1}+b \bmod p^{k}$. Note that $\mu-1=$ 
$(p-1) p^{k-1}-1 \geq k$ if either $k \geq 1$ when $p$ is odd or $k \geq 3$ when $p=2$. So, $x_{t+1} \equiv a(c p)^{\mu-1}+b \equiv b \bmod p^{k}$.

Using this lemma, we can prove the following theorem which will list all possible period lengths of sequences with modulus $p^{k}$ defined by the recursion (3) and containing 0 with modulus $p$.

TheOREM 11. Let $p$ be a prime and $k \geq 2$ be a positive integer. Let $a, b$ and $x_{0}$ be integers, and let the sequence $X\left(x_{0} ; a, b\right): x_{0}, x_{1}, \ldots$ with modulus $p^{k}$ be defined by the recursion (3). Moreover, suppose the sequence $X\left(x_{0} ; a, b\right)$ with modulus $p$ contains 0 .

(A) If $a \equiv 0 \bmod p$ and either $b \equiv 0 \bmod p$ or $x_{0} \equiv 0 \bmod p$, then $L_{G}\left(x_{0} ; a, b ; p^{k}\right)=1$.

(B) If $\operatorname{gcd}(a, p)=1$ and $b \equiv 0 \equiv x_{0} \bmod p$, then $L_{G}\left(x_{0} ; a, b ; p^{k}\right)=1$ except for the case $p=2=k$ and $b \equiv 2 \bmod 4$. For this exceptional case, $L_{G}\left(x_{0} ; a, b ; 4\right)=2$.

(C) If $\operatorname{gcd}\left(a b\left(x_{0}^{2}-b x_{0}+a\right), p\right)=1$ and $b^{2}+4 a \equiv 0 \bmod p$, then $L_{G}\left(x_{0} ; a, b ; p^{k}\right)=p-1$.

(D) If $p$ is odd, $\operatorname{gcd}\left(a b\left(b^{2}+4 a\right), p\right)=1, x_{0} \equiv b / 2 \bmod p$, and the order $\mathfrak{o}\left(m_{f}\right)$ of the polynomial $m_{f}(x)=x^{2}+\left(b^{2} / a+2\right) x+1$ in $\operatorname{GF}(p)[x]$ is even, then $L_{G}\left(x_{0} ; a, b ; p^{k}\right)=\mathfrak{o}\left(m_{f}\right)-1$.

(E) If $\operatorname{gcd}\left(a b\left(b^{2}+4 a\right)\left(x_{0}^{2}-b x_{0}+a\right), p\right)=1, x_{0} \not \equiv b / 2 \bmod p$ for $p \neq 2$, and the order $\mathfrak{o}\left(M_{f}\right)$ of the polynomial $M_{f}(x)=x^{2}-\left(2+\left(b^{2}+a\right) /\left(x_{0}^{2}-\right.\right.$ $\left.\left.b x_{0}+a\right)\right) x+1$ in $\operatorname{GF}(p)[x]$ divides $\mathfrak{o}\left(m_{f}\right)$, then $L_{G}\left(x_{0} ; a, b ; p^{k}\right)=\mathfrak{o}\left(m_{f}\right)-1$ except for the case $p=2=k$ and $a \equiv 1 \bmod 4$. For this exceptional case, $L_{G}\left(x_{0} ; a, b ; 4\right)=4$.

Proof. (A) From Lemma $1(\mathrm{~A}), L_{G}\left(x_{0} ; a, b ; p\right)=1$. Since the sequence $X\left(x_{0} ; a, b\right)$ with modulus $p$ contains $0, \operatorname{gcd}(b, p)=1 \operatorname{implies} x_{0} \equiv 0 \bmod p$ and so, $x_{1} \equiv b \bmod p^{k}$ by Lemma 10 and the fact that $a x_{0} \equiv 0 \bmod 4$ when $p=2$. In this case, it suffices to consider $X(b ; a, b)$ with modulus $p^{k}$. Since $\operatorname{gcd}(b, p)=1, L_{G}\left(b ; a, b ; p^{k}\right)=1$ by Theorem $5(\mathrm{~A})$ and so $L_{G}\left(x_{0} ; a, b ; p^{k}\right)$ $=1$. Now, suppose $b \equiv 0 \bmod p$. From Lemma $1(\mathrm{~A})$ again, $x_{n} \equiv b \equiv 0$ $\bmod p$. Then this case follows from Lemma 10 except for the case $p^{k}=4$. For this exception, $a b^{2-1}+b \equiv b \bmod 4$ since $\phi(4)=2$ and $a \equiv 0 \equiv b \bmod 2$. So, $x_{n} \equiv b \bmod 4$ for all $n \geq 1$. Therefore, $L_{G}\left(x_{0} ; a, b ; 4\right)=1$.

From now on, let $\operatorname{gcd}(a, p)=1$. Then $X\left(x_{0} ; a, b\right)$ with modulus $p^{k}$ is purely periodic.

(B) From Lemma 1(B), $L_{G}\left(x_{0} ; a, b ; p\right)=1$. Then the case follows from Lemma 10 except for $p^{k}=4$. If $b \equiv 2 \bmod 4$, then $x_{n} \equiv 0 \bmod 2$ and $x_{n} \not \equiv x_{n+1} \bmod 4$ for all $n \geq 0$, because of $x_{0} \equiv 0 \bmod 2$ and $\operatorname{gcd}(a, 2)=1$. So, $L_{G}\left(x_{0} ; a, b ; 4\right)=2$ if $b \equiv 2 \bmod 4$. If $b \equiv 0 \bmod 4$, then $x_{n} \equiv x_{0} \bmod 4$ for all $n \geq 0$. Hence, $L_{G}\left(x_{0} ; a, b ; 4\right)=1$ if $b \equiv 0 \bmod 4$. 
(C) Note that $p \neq 2$ in this case. From Lemma 1(E)(2), the sequence $X\left(x_{0} ; a, b\right)$ with modulus $p$ contains 0 and so $L_{G}\left(x_{0} ; a, b ; p\right)=p-1$. Then this case follows from Lemma 10.

(D) This case follows from Lemma $1(\mathrm{~F})(2)$ and Lemma 10 immediately.

(E) This case follows from Lemma $1(\mathrm{~F})(4)$ and Lemma 10 except for the case $p=2=k$. We now consider the exceptional case. Since $\operatorname{gcd}(a b, 2)$ $=1$, we have $a \equiv 1 \equiv b \bmod 2$. So, $a \equiv 1,3 \bmod 4, b \equiv 1,3 \bmod 4$, and $x_{0} \equiv 0,1,2,3 \bmod 4$. By checking all possible cases, $L_{G}\left(x_{0} ; a, b ; 4\right)=2$ if $a \equiv 3 \bmod 4$, and $L_{G}\left(x_{0} ; a, b ; 4\right)=4$ if $a \equiv 1 \bmod 4$. Finally, note that $m_{f}(x)=x^{2}+x+1$ in $\operatorname{GF}(2)[x]$ has order 3 . This completes the proof of this theorem.

Let $m, a, b$ and $x_{0}$ be integers with $m>0$. Let the sequence $X\left(x_{0} ; a, b\right)$ with modulus $m$ be defined by the recursion (3). Huber [9] showed that if $m$ is square free, then $X\left(x_{0} ; a, b\right)$ with modulus $m$ has the maximal period length if and only if the polynomial $f(x)=x^{2}-b x-a$ is an IMP (abbreviated for inversive maximal period) polynomial in $\operatorname{GF}(p)[x]$ for every prime divisor $p$ of $m$. So, if $m$ is square free and $X\left(x_{0} ; a, b\right)$ with modulus $m$ has the maximal period length, then its period length is $m$. This is no more true if $m$ is not square free. In fact, if $m=p_{1} \ldots p_{s-1} p_{s}^{r_{s}} \ldots p_{t}^{r_{t}}$ is the prime factorization of $m$, where $p_{1}, \ldots, p_{t}$ are distinct primes and $r_{s}, \ldots, r_{t}$ are positive integers greater than 1 , then the sequence $X\left(x_{0} ; a, b\right)$ with modulus $m$ has the maximal period length if and only if $f(x)=x^{2}-b x-a$ is an IMP polynomial in $\operatorname{GF}\left(p_{i}\right)[x]$ for all $1 \leq i<s$, and $a, b, x_{0}$ and $p_{j}^{r_{j}}$ satisfy the conditions of Corollary 9 for all $s \leq j \leq t$.

Acknowledgements. The author is sincerely grateful to Prof. Dr. H. Niederreiter of the Institute of Information Processing, Austrian Academy of Sciences, Austria, for many helpful discussions and useful comments.

\section{References}

[1] W.-S. Chou, On inversive maximal period polynomials over finite fields, Appl. Algebra Engrg. Comm. Comput. 6 (1995), 245-250.

[2] - , The period lengths of inversive pseudorandom vector generators, Finite Fields Appl. 1 (1995), 126-132.

[3] J. Eichenauer and J. Lehn, A non-linear congruential pseudorandom number generator, Statist. Hefte 27 (1986), 315-326.

[4] J. Eichenauer, J. Lehn and A. Topuzoğlu, A nonlinear congruential pseudorandom number generator with power of two modulus, Math. Comp. 51 (1988), $757-759$

[5] J. Eichenauer-Herrmann, Inversive congruential pseudorandom numbers: a tutorial, Internat. Statist. Rev. 60 (1992), 167-176.

[6] - Construction of inversive congruential pseudorandom number generators with maximal period length, J. Comput. Appl. Math. 40 (1992), 345-349. 
[7] J. Eichenauer-Herrmann and A. Topuzoğlu, On the period length of congruential pseudorandom number sequences generated by inversions, ibid. 31 (1990), 87-96.

[8] M. Flahive and H. Nied erreiter, On inversive congruential generators for pseudorandom numbers, in: Finite Fields, Coding Theory, and Advances in Communications and Computing, G. L. Mullen and P. J.-S. Shiue (eds.), Marcel Dekker, New York, 1992, 75-80.

[9] K. Huber, On the period length of generalized inversive pseudorandom generators, Appl. Algebra Engrg. Comm. Comput. 5 (1994), 255-260.

[10] H. Niederreiter, Finite fields and their applications, in: Contributions to General Algebra, Vol. 7, Vienna, 1990, Teubner, Stuttgart, 1991.

[11] - Random Number Generation and Quasi-Monte Carlo Methods, SIAM, Philadelphia, PA, 1992.

[12] -, Pseudorandom vector generation by the inversive method, ACM Trans. Modeling \& Computer Simulation 4 (1994), 191-212.

INSTITUTE OF MATHEMATICS

ACADEMIA SINICA

NANKANG, TAIPEI 11529

TAIWAN, ROC

E-mail: MACWS@CCVAX.SINICA.EDU.TW

Received on 13.4.1994

and in revised form on 15.9.1994 